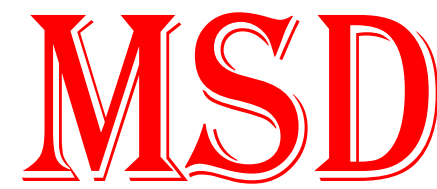

Medical Science and Discovery ISSN: 2148-6832

\section{COVID-19 infection in patients receiving hemodialysis in Athens: Findings, experience, and outcome from a single Dialysis Unit}

\author{
Ioannis Griveas ${ }^{1 *}$, Antonios Schoinas ${ }^{1}$, Anthi Balitsari ${ }^{1}$, Gerasimos \\ Asimakopoulos ${ }^{1}$, Evaggelos Pratilas ${ }^{1}$ \\ 1 Dept. of Nephrology, Army Share Fund Hospital of Athens 417 NIMTS, Greece \\ * Corresponding Author: Ioannis Griveas E-mail: giannisgriv@ hotmail.com
}

\section{ABSTRACT}

Objective: Our Nephrology Department during the spring period on the first wave of COVID-19 was the referral Dialysis Unit for Covid-19 positive HD patients in the district area of Athens, Greece. This study aims to report the characteristics, rates, and outcomes of all patients affected by infection with SARS-CoV-2 undergoing HD and treated under our care. 22 Covid-19 positive HD patients were treated under the care of our facility during the period 8 April 2020-17 June 2020. 16 patients were symptomatic at admission and 13 patients were admitted with or developed during their stay pleural effusions. 12 patients ( 8 male) of our group died during their hospitalization. The mean age of our patients was 74.5 years. It has to be pointed out that 13 patients were over 75 years old. Mean age was higher in those who died compared with those who were discharged with double negative Covid-19 tests (79 vs 74,5 years old respectively). It seems that despite the fact that the immune response of this population has not been clearly clarified, age comorbidities and above all end-stage renal disease by its self is a significant and unpredictable risk factor for clinical outcome of HD patients with COVID-19 infection.

Keywords: Haemodialysis, COVID-19, nephrology, outcome, SARS-CoV-2

\section{INTRODUCTION}

In December 2019 a new strain of coronavirus, officially named severe acute respiratory syndrome coronavirus 2 (SARS-Cov-2), was first isolated from three patients with coronavirus disease 2019 (covid-19) by the Chinese Center for Disease Control and Prevention connected to the cluster of acute respiratory illness cases from Wuhan, China $(1,2)$. On 30 January 2020, the World Health Organization declared that the outbreak of SARS-Cov-2 constituted a public health emergency of international concern.

End-Stage-Renal Disease patients under hemodialysis (HD) are at increased risk for coronavirus disease 2019 (COVID-19) and its complications, owing to the presence of multiple comorbid conditions. Patients receiving kidney replacement therapy are a vulnerable population as those receiving dialysis are usually older with significant comorbidity and have also impaired immune responses (3) and require regular attendance at a healthcare facility. The logistical aspects within a dialysis facility further increase the risk of disease transmission (4). There are data in the literature that suggesting a more severe disease course in patients with chronic kidney disease (CKD) (5). Still outcomes in End-Stage-Renal Disease patients under HD are unclear, with small case series suggesting a milder course (6). On the other hand, HD patients are usually old and affected by several comorbidities such as diabetes mellitus and hypertension that are known to be associated with a high risk of poor outcomes in patients with coronavirus disease 2019 (COVID-19).

The above notifications clearly imply that although there have been a number of published recommendations on how to mitigate the spread of SARS-CoV-2 in dialysis units $(7,8,9,10,11)$ at the same time there is a lack of data regarding clinical course and outcome in patients receiving HD following infection with SARS-CoV-2. 
Our Nephrology Department during the spring period on the first wave of COVID-19 was the referral Dialysis Unit for Covid-19 positive HD patients in the district area of Athens, Greece. This study aims to report the characteristics, rates, and outcomes of all patients affected by infection with SARSCoV-2 undergoing HD and treated under our care

\section{MATERIAL and METHODS}

This is an observational study. Our Dialysis Unit has been assigned as a referral unit for Covid-19 positive HD patients. We registered all the data regarding the clinical course of our patients' population. Age, the primary cause of end-stage renal disease, weight, clinical presentation, HD history, outcome, days of hospitalization.

\section{RESULTS}

The 22 Covid-19 positive HD patients were treated under the care of our facility during the period 8 April 2020-17 June 2020. 16 patients were symptomatic at admission and 13 patients were admitted with or developed during their stay pleural effusions. 12 patients (8 male) of our group died during their hospitalization. 3 out of 12 were admitted to the Intensive Care Unit (ICU). 6 patients were septic, 4 had respiratory failure, and 2 developed cardiovascular events. 14.5 days were the mean hospitalization days (range: 1-38 days) for the deceased ones.

The 2 out of 3 patients that were admitted to ICU had quick deterioration, incubated, and stayed in ICU for 48 hours. The third one with severe comorbidities (multiple myeloma, cancer of bladder) developed respiratory failure after 8 days of hospitalization, incubated, became septic, and died after 20 days in ICU.

The mean age of our patients was 74.5 years. It has to be pointed out that 13 patients were over 75 years old. Mean age was higher in those who died compared with those who were discharged with double negative Covid-19 tests (79 vs 74,5 years old respectively). The median dialysis vintage for our patients was 63 months and for the deceased ones was 89 months. The average weight of our patients was $69 \mathrm{~kg}$. The weight of deceased patients was $63 \mathrm{~kg}$. 11 out of 22 patients and 5 out of 12 deceased patients were diabetic. 14 patients were hypertensive and 16 had an official cardiovascular background. 10 out of total 22 patients under our care discharged after 43 days of hospitalization ( range:35-56 days).

\section{DISCUSSION}

There is currently a lack of data on how SARS-CoV-2 affects patients receiving HD. Our morbidity rates are higher than the average number from the literature. In Spain and Italy, mortality rates were $30.5 \%$ and $23.6 \%$ respectively $(12,13)$. $23.6 \%$ was also recorded in the Alsace region of France, where the vast majority of deceased patients died 2 weeks after the onset of the symptoms (14). Furthermore, in Northern Italy the mortality rates of COVID-19 were calculated as $24.6 \%$ for HD patients (15). Similarly, a study of 59 consecutive patients testing positive from Columbia University in the United States found a mortality rate of $31 \%$ (18 patients)(16).
The mean age of our study population is quite high, with significant comorbidities that co-exist, a fact which explains the outcome. A report from the COVID-19 registry of the Spanish Society of Nephrology reported a mortality of $23 \%$ in 868 patients with SARS-CoV-2 infection. Mortality was also associated with increasing age in this cohort (17). A study from a single urban center in the United Kingdom showed much higher rates of transmission with over $20 \%$ of their HD patients affected within 6 weeks of detection of their first case. Similar mortality rates were demonstrated in this series with $20.3 \%$ of patients dying during follow-up, again with increasing age being associated with a higher likelihood of mortality (18).

In the general population, several comorbid conditions such as diabetes, hypertension, and obesity have consistently been linked to increased risk of death with COVID-19 (19). The majority of our patients were old, diabetic with hypertension and cardiovascular background. It is crucial to point out that for patients requiring $\mathrm{HD}$, the risk attributable to the requirement for HD is such a strong risk factor for death that it is challenging to identify subgroups of dialysis recipients at the highest risk. At the same time, the immune response of this group of patients is not clearly understood and known especially in conjunction with the behavior of Covid-19 infection.

Our data confirm reports from recent literature that the mortality in HD patients is much higher than in the general population, $13 \%$ vs $4 \%$ respectively $(4 \%$ is the official mortality in the general COVID-19 population reported in China). Nevertheless, although the infection may be fatal, COVID-19 symptoms in dialysis patients in many cases seem to be less aggressive, a fact that we also noticed in some of our patients. It has been postulated that HD patients may be relatively protected from violent cytokine storms by their impaired immune systems (20).

The number of patients in our cohort who required intensive care is perhaps lower than might be expected. However, all 3 patients that were admitted to ICU died. They had severe comorbidities, but one of them remained 20 days in ICU before passed away.

The question of whether a patient dies by COVID-19 or with COVID-19 is misleading especially in HD population and perhaps it is going to remain unanswered. However HD patients are such complex and fragile patients and a violent infection such as COVID-19, that mainly targets lungs and heart, already very compromised for many years of dialysis, brings a very high risk of death, even if the infection itself is not the final cause $(21,22)$.

Another issue, that is not referred to our patients, is dialysis adequacy for COVID-19 positive HD patients. Despite the heavy schedule of our medical-nursing personnel, all of our patients received HD sessions 3 times a week (no exceptions) for 3 to 4 hours per session. In 2 cases where tunneled lines were not performing well, they were replaced. This is the reason that we did not have any death from hyperkalemia. A question has raised in patients where dialysis frequency was reduced to minimize infection risk: how many victims of other diseases will be indirectly related to the coronavirus pandemic? (20). 


\section{CONCLUSION}

As a conclusion, our data provide clues regarding our experience of caring for HD patients with COVID-19. Mortality was high. It seems that despite the fact that the immune response of this population has not been clarified, age, comorbidities and above all end-stage renal disease by its self is a significant and unpredictable risk factor for the clinical outcome of HD patients with COVID-19 infection.

Author contributions: IG, AS, GA, EP; Patient examination and therapy, Literature search and study design, IG; Writing article and revisions

Conflict of interest: The authors declared no potential conflicts of interest with respect to the research, authorship, and/or publication of this article. This research did not receive and a specific grant from funding agencies in the public, commercial, or not-for-profit sectors.

Ethical issues: All authors declare originality of research.

\section{REFERENCES}

1. Zhu N, Zhang D, Wang W, et al. A Novel Coronavirus from Patients with Pneumonia in China, 2019. N Engl J Med 2020;382:727-733.

2. Gralinski LE, Menachery VD. Return of the Coronavirus: 2019-nCoV. Viruses 2020;12:135.

3. Kato S, Chmielewski M, Honda $\mathrm{H}$, et al. Aspects of immune dysfunction in end-stage renal disease. Clin J Am Soc Nephrol. 2008;3:1526-33.

4. Kliger AS, Cozzolino M, Jha V, et al. Managing the COVID-19 pandemic: international comparisons in dialysis patients. Kidney Int. 2020;98:12-16.

5. Henry BM, Lippi G. Chronic kidney disease is associated with severe coronavirus disease 2019 (COVID-19) infection. Int Urol Nephrol. $2020 ; 52: 1193-1194$

6. Wang R, Liao $\mathrm{C}, \mathrm{He} \mathrm{H}$, et al. COVID-19 in hemodialysis patients: report of 5 cases. Am J Kidney Dis 2020;76(1):141-143.

7. Basile C, Combe C, Pizzarelli F, et al. Recommendations for the prevention, mitigation and containment of the emerging SARS-CoV-2 (COVID-19) pandemic in haemodialysis centres. Nephrol Dialysis Transplant. 2020;35:737-741.

8. Ikizler TA, Kliger AS. Minimizing the risk of COVID-19 among patients on dialysis. Nat Rev Nephrol. 2020;16:311-313.
9. Meijers B, Messa P, Ronco C. Safeguarding the maintenance hemodialysis patient population during the coronavirus disease 19 pandemic. Blood Purif. 2020;49:259-264.

10. Kliger AS, Cozzolino M, Jha V, Harbert G, Ikizler TA. Managing the COVID-19 pandemic: international comparisons in dialysis patients Kidney Int. 2020; 98:12-16.

11. UK Renal Association. PPE and use of masks by dialysis patients 2020 [Available from: https://renal.org/renal-association-statement-ppe-usemasksdialysis-patients/\#layout-secondary-nav

12. Goicoechea M, Camara LAS, Macias N, et al. COVID-19: Clinical course and outcomes of 36 hemodialysis patients in Spain. Kidney Int. 2020;98:27-34.

13. Milia VL, Bacchini G, Bigi MC, et al. COVID-19 outbreak in a large hemodialysis center in Lombardy, Italy. Kidney Int Rep. 2020;5:10951099.

14. Keller N, Chantrel F, Krummel T, et al. Impact of first-wave corona virus disease 2019 infection in patients on haemodialysis in Alsace: The observational COVIDAL study. Neprol Dial Transplant. 2020;35:13381345 .

15. Manganaro M, Baldovino S. First considerations on the SARSCoV-2 epidemic in the dialysis units of Piedmont and Aosta Valley, Northern Italy. J Nephrol. 2020;10:1-3

16. Valeri AM, Robbins-Juarez SY, Stevens JS, et al. Presentation and outcomes of patients with ESKD and COVID-19. J Am Soc Nephrol. 2020;31:1409-15

17. Sánchez-Álvarez JE, Pérez Fontán M, Jiménez Martín C, et al Situación de la infección por SARS-CoV-2 en pacientes en tratamiento renal sustitutivo. Informe del Registro COVID-19 de la Sociedad Española de Nefrología (SEN). Nefrología. 2020;40:272-78.

18. Corbett RW, Blakey S, Nitsch D, Loucaidou M, McLean A, Duncan N, et al. Epidemiology of COVID-19 in an Urban Dialysis Center. J Am Soc Nephrol. 2020;31:1815-23.

19. Docherty AB, Harrison EM, Green CA, et al. Features of 20133 UK patients in hospital with covid-19 using the ISARIC WHO clinical characterisation protocol: prospective observational cohort study. BMJ. 2020;369:1985

20. Ma Y, Diao B, Lv X, et al. 2019 novel coronavirus disease in hemodialysis (HD) patients: Report from one HD center in Wuhan China. medRxiv 2020

21. Guan WJ, Ni ZY, Hu Y et al (2020) China medical treatment exper group for Covid-19. Clinical characteristics of coronavirus 2019 disease in China. N Engl J Med 2020;30:1708-1720.

22. Naicker S, Yang CW, Hwang SJ, Liu BC, Chen JH, Jha V (2020). The novel coronavirus 2019 epidemic and kidneys. Kidney Int 2020;97:824828

Copyright (C) 2021 The Author(s); This is an open-access article distributed under the terms of the Creative Commons Attribution License (http://creativecommons.org/licenses/by/4.0), (CC BY NC) which permits unrestricted use, distribution, and reproduction in any medium, provided the original work is properly cited. International journal of Medical Science and Discovery. 